\title{
A radiobiological study of the schemes with a low number of fractions in high-dose-rate brachytherapy as monotherapy for prostate cancer
}

\author{
Damián Guirado, PhD',2,3, Samuel Ruiz-Arrebola, MSc4, Ana M. Tornero-López, PhD5, Jose M. de la Vega, PhD1.2, \\ Pedro J. Prada, PhD 4 , Antonio M. Lallena, PhD²,6 \\ "Unidad de Radiofisica, Hospital Universitario Clínico "San Cecilio", E-180l6 Granada, Spain, 2Instituto de Investigación Biosanitaria \\ (ibs. GRANADA), Complejo Hospitalario Universitario de Granada/Universidad de Granada, Granada, Spain, ${ }^{3}$ CIBER de Epidemiología \\ y Salud Pública (CIBERESP), Granada, Spain, "Servicio de Oncología Radioterápica, Hospital Universitario "Marqués de Valdecilla" \\ Santander, Spain, ${ }^{5}$ Servicio de Radiofisica y Protección Radiológica, Hospital Universitario Dr. Negrín, Las Palmas de Gran Canaria, Spain, \\ 'Departamento de Física Atómica, Molecular y Nuclear, Universidad de Granada, Granada, Spain
}

\begin{abstract}
Purpose: Schemes with high doses per fraction and small number of fractions are commonly used in high-dose-rate brachytherapy (HDR-BT) for prostate cancer. Our aim was to analyze the differences between published clinical results and the predictions of radiobiological models for absorbed dose required in a single fraction monotherapy HDR-BT.

Material and methods: Published HDR-BT clinical results for low- and intermediate-risk patients with prostate cancer were revised. For 13 clinical studies with 16 fractionation schedules between 1 and 9 fractions, a dose-response relation in terms of the biochemical control probability (BC) was established using Monte Carlo-based statistical methods.

Results: We obtained a value of $\alpha / \beta=22.8 \mathrm{~Gy}$ (15.1-60.2 Gy) (95\% CI) much larger than the values in the range 1.5-3.0 Gy that are usually considered to compare the results of different fractionation schemes in prostate cancer radiotherapy using doses per fraction below $6 \mathrm{~Gy}$. The doses in a single fraction producing $\mathrm{BC}=90 \%$ and $95 \%$ were 22.3 Gy (21.5-24.2 Gy) and 24.3 Gy (23.0-27.9 Gy), respectively.

Conclusions: The $\alpha / \beta$ obtained in our analysis of $22.8 \mathrm{~Gy}$ for a range of dose per fraction between 6 and $20.5 \mathrm{~Gy}$ was much greater than the one currently estimated for prostate cancer using low doses per fraction. This high value of $\alpha / \beta$ explains reasonably well the data available in the region of high doses per fraction considered.
\end{abstract}

J Contemp Brachytherapy 2020; 12, 2: 193-200 DOI: https://doi.org/10.5114/jcb.2020.94492

Key words: HDR brachytherapy, prostate cancer, monotherapy.

\section{Purpose}

Apart from prostatectomy, radiotherapy is the most used therapeutic option for localized prostate cancer, with external beam radiotherapy (EBRT), brachytherapy (BT), or both techniques applied. Since mid-1990s, highdose-rate-brachytherapy (HDR-BT) started to be used as monotherapy $[1,2,3]$ and demonstrated to be an effective therapy, with precise dose distribution, reduced total treatment time and cost as well as increased patient comfort $[4,5]$.

According to evidences accumulated in recent years, prostate cancer appears to be particularly sensitive to fractionation, much more than the healthy tissues also affected by radiotherapy. This sensitivity appears in a low value of the $\alpha / \beta$ parameter of the linear-quadratic (LQ) model, where to calculate the equivalence between different fractionation schemes used in clinical practice, a value of $\alpha / \beta$ between 1.5 and 3.0 Gy is usually considered [6]. All of this has led to the proliferation of clinical studies and applications of hypofractionation in HDR-BT for prostate cancer, as in case of EBRT $[7,8]$.

Recently, the possibility of single fraction monotherapy HDR-BT comparable to treatments involving several fractions has been explored. Mavroidis et al. [9] estimated the dose of that single fraction and found that a dose between 19.2 and 19.7 Gy is required to produce a tumor control equal to or greater than that of a scheme with 4 fractions, 9.5 Gy each, specifically $95.9 \%$ to $97.8 \%$ vs. $93.5 \%$ to $97.8 \%$. However, for the latter scheme, a biochemical control probability (BC) at 5 years between $91 \%$ and $97 \%$ was obtained for low- and intermediate-risk patients [10,11,12], 
while for the same groups of patients, $66 \pm 6 \%$ and $82 \pm 3 \%$, both at $95 \%$ confidence interval (CI) for single doses of 19.0 Gy [13] and 20.5 Gy [14], respectively, were found.

These differences between predictions and clinical results could be due to the fact that the LQ model is not appropriate for high doses per fraction, requiring some modification in order to be employed in this context $[15,16]$, or the value of the $\alpha / \beta$ parameter obtained in the region of low doses per fraction is not valid when these doses per fraction are high [17].

In this work, we analyzed the discrepancies between the published clinical results and the predictions of the radiobiological models to determine the value of absorbed dose required in an extreme, single fraction, hypofractionation regime in HDR-BT monotherapy, leading to a biochemical failure rate similar to that of the most widely used regimes. In order to do that, we analyzed the published HDR-BT clinical results, establishing a dose-response relation in terms of $\mathrm{BC}$ by means of statistical methods based on a Monte Carlo procedure.

\section{Material and methods}

\section{Clinical data analyzed}

The clinical data analyzed in the present work were collected after a systematic search in PubMed in January 2019, with key terms including "high-dose-rate brachytherapy" or "HDR brachytherapy", "prostate cancer", and "monotherapy", followed by a more detailed search within the bibliography found in the first step, and by looking for prospective or retrospective clinical studies verifying the following conditions:

1. HDR ${ }^{192}$ Ir interstitial BT was the only radiotherapy technique used, without EBRT.

2. The endpoint considered was BC obtained as 1 minus prostate-specific antigen (PSA) failure, defined as nadir plus $2 \mathrm{ng} / \mathrm{ml}$, according to [18].

3. The BC at 5 years for the fractionation schemes considered was explicitly provided. Studies in which this endpoint may be obtained indirectly from their outcomes were not considered.

4. Patients of low- and/or intermediate-risk were included. BC values in both groups together or separately were considered. Studies involving high-risk patients were considered only if BC was applied for low- or intermediate-risk patients, separately.

Table 1 summarizes characteristics of the 13 clinical studies $[10,11,12,13,14,19,20,21,22,23,24,25,26]$ verifying the conditions mentioned above. They include 16 different fractionation schedules, with a number of fractions varying between 1 and 9, for a total of 22 empirical data sets. Table 1 includes dosimetric details of these fractions, the risk group, and the BC at 5 years, with the $95 \% \mathrm{CI}$ when quoted by the study authors.

\section{Dose response model}

In this study, BC was described by means of [27]:

$\mathrm{BC}=\left[1+\left(\frac{D_{50}}{D}\right)^{4 \gamma}\right]^{-1}$. where

$$
\gamma=\left.D \frac{\mathrm{dBC}}{\mathrm{d} D}\right|_{\max }=\left.D \frac{\mathrm{dBC}}{\mathrm{d} D}\right|_{D_{50}}
$$

is the maximum normalized dose-response gradient, and $D_{50}$ is the total dose needed to achieve $\mathrm{BC}=50 \%$.

To compare different HDR-BT schemes, we considered the biologically effective dose [28]:

$\operatorname{BED}(D, d)=D\left(1+\frac{d}{\alpha / \beta}\right)$.

Here $D$ is the total dose, $d$ is the dose per fraction, and the $\alpha / \beta$ LQ parameter allows quantifying the sensitivity of fractionation. The equivalent dose $\mathrm{EQD}_{2}$ is defined as the total dose imparted in a scheme with 2 Gy per fraction that produces the same biological effect than another scheme:

$\operatorname{BED}\left(\mathrm{EQD}_{2}, 2 \mathrm{~Gy}\right)=\operatorname{BED}(D, d)$.

Using equation (2) we have:

$\mathrm{EQD}_{2}=D \frac{d+\alpha / \beta}{2 \mathrm{~Gy}+\alpha / \beta}$.

The total treatment time of the various schemes analyzed was not considered and its effect is not included in equations (2) and (4).

It is possible to establish a dose-response relationship including all the fractionation schemes, in which the total dose $D$ in equation (1) is substituted by the equivalent dose $\mathrm{EQD}_{2}$, and using equation (4) we have:

$\mathrm{BC}=\left[1+\left(\frac{D_{50}}{\mathrm{EQD}_{2}}\right)^{4 \gamma}\right]^{-1}=\left[1+\left(\frac{D_{50}}{D} \frac{2 \mathrm{~Gy}+\alpha / \beta}{d+\alpha / \beta}\right)^{4 \gamma}\right]^{-1}$.

\section{Statistical methods}

The $\mathrm{BC}$ data shown in Table 1 were used to determine the free parameters $D_{50}, \gamma$, and $\alpha / \beta$ in equation (5), using the Monte Carlo procedure described below.

1. For each clinical study in Table 1, two parameters were calculated. The first one, $\mu$, was chosen as the average $\mathrm{BC}$ provided in the study (column \#7 in the Table 1). In three studies of [12], where $95 \%$ CI (column \#8) is markedly asymmetric with respect to the average value, $\mu$ was chosen as the center of the CI.

The second parameter, $\sigma$, was calculated as:

$\sigma=\frac{1}{3.92}\left(\mathrm{BC}_{\text {high }}-\mathrm{BC}_{\mathrm{low}}\right)$

for the studies providing 95\% CI [(BC) $\left.)_{\mathrm{high}^{\prime}}(\mathrm{BC})_{\mathrm{low}}\right]$, and its variation with the number of patients, $n$, was fitted with the function $\sigma=a n^{-\frac{1}{2}}$, with $a$ being the fitting parameter. The data obtained are shown in Figure 1 with symbols and the curve fitted to all these data is the dashed one. If the data shown by open squares, considered as outliers, are not included in the fit, the solid curve is obtained. As both fitting curves produce the same final results, the $\sigma$ values considered in our analysis were calculated with equation (6) or obtained with the dashed fitting curve depending on availability of $95 \% \mathrm{CI}$.

The value $3.92=2 \times 1.96$ in equation (6) is due to the fact that CI corresponds to $95 \%$ probability. The $\mu$ and $\sigma$ 
Table 1. Summary of the prostate cancer HDR-BT monotherapy data analyzed. The number of sessions, the dose per fraction, $d$, the total dose, $D$, the number of patients, the average $\mathrm{BC}$ at 5 years endpoint, and its $95 \% \mathrm{Cl}$ are given for low (L)- and intermediate (I)-risk patients. The last two columns show the $\mu$ and $\sigma$ considered in the fitting procedure described in the text. For the study of [21], the total dose and dose per fraction averages were considered. The study [22] was included despite involving three high-risk patients. The BC of [23] is for 6 years

\begin{tabular}{|c|c|c|c|c|c|c|c|c|c|}
\hline \multirow[t]{2}{*}{ Reference } & \multicolumn{3}{|c|}{ Fractionation schedule } & \multirow[t]{2}{*}{ Risk } & \multirow{2}{*}{$\begin{array}{c}\text { \# of } \\
\text { patients }\end{array}$} & \multicolumn{2}{|c|}{$\mathrm{BC}$ at 5 years $(\%)$} & \multirow[t]{2}{*}{$\mu$} & \multirow[t]{2}{*}{$\sigma$} \\
\hline & $n$ & $d$ (Gy) & $D$ (Gy) & & & Average & $95 \% \mathrm{Cl}$ & & \\
\hline \multirow{2}{*}{ [10] } & 4 & 9.5 & 38.0 & \multirow{2}{*}{$\mathrm{L} / \mathrm{I}$} & 171 & 91.0 & & 91.0 & 2.6 \\
\hline & 6 & 7.0 & 42.0 & & 77 & 87.0 & & 87.0 & 3.8 \\
\hline \multirow{2}{*}{ [11] } & \multirow{2}{*}{4} & \multirow{2}{*}{9.5} & \multirow{2}{*}{38.0} & $\mathrm{~L}$ & 196 & 94.0 & & 94.0 & 2.4 \\
\hline & & & & । & 81 & 92.0 & & 92.0 & 3.7 \\
\hline \multirow{3}{*}{ [12] } & 4 & 9.5 & 38.0 & \multirow{3}{*}{$\mathrm{L} / \mathrm{I}$} & 319 & 97.0 & {$[93.5,98.7]$} & 96.1 & 1.3 \\
\hline & 2 & 12.0 & 24.0 & & 79 & 87.0 & {$[68.0,95.1]$} & 81.6 & 6.9 \\
\hline & 2 & 13.5 & 27.0 & & 96 & 90.0 & {$[63.8,97.6]$} & 80.7 & 8.6 \\
\hline [13] & 1 & 19.0 & 19.0 & $\mathrm{~L} / \mathrm{I}$ & 60 & 66.0 & {$[60.0,72.0]$} & 66.0 & 3.1 \\
\hline [14] & 1 & 20.5 & 20.5 & $\mathrm{~L} / \mathrm{I}$ & 60 & 82.0 & {$[79.0,85.0]$} & 82.0 & 1.5 \\
\hline \multirow{2}{*}{ [19] } & \multirow{2}{*}{9} & \multirow{2}{*}{6.0} & \multirow{2}{*}{54.0} & L & 15 & 85.0 & {$[66.0,100.0]$} & 85.0 & 8.7 \\
\hline & & & & I & 29 & 93.0 & {$[83.0,100.0]$} & 93.0 & 4.3 \\
\hline [20] & 6 & 6.5 & 39.0 & I & 284 & 94.4 & {$[90.1,98.7]$} & 94.4 & 2.2 \\
\hline \multirow{4}{*}{ [21] } & \multirow{4}{*}{3} & 10.0 & 30.0 & \multirow{4}{*}{$\mathrm{L} / \mathrm{I}$} & 19 & \multirow{4}{*}{85.1} & \multirow{4}{*}[72.5,94.5]{} & \multirow{4}{*}{85.1} & \multirow{4}{*}{5.6} \\
\hline & & 10.5 & 31.5 & & 19 & & & & \\
\hline & & 11.0 & 33.0 & & 19 & & & & \\
\hline & & 11.5 & 34.5 & & 22 & & & & \\
\hline [22] & 3 & 15.0 & 45.0 & $\mathrm{~L} / \mathrm{I}$ & 77 & 96.7 & & 96.7 & 3.8 \\
\hline [23] & 6 & 7.25 & 43.5 & $\mathrm{~L} / \mathrm{I}$ & 448 & 98.6 & {$[96.9,99.4]$} & 98.6 & 0.6 \\
\hline \multirow{2}{*}{ [24] } & \multirow{2}{*}{3} & \multirow{2}{*}{11.5} & \multirow{2}{*}{34.5} & L & 198 & 96.1 & {$[92.4,99.3]$} & 96.1 & 1.8 \\
\hline & & & & I & 135 & 96.1 & {$[92.1,99.9]$} & 96.1 & 2.0 \\
\hline [25] & 2 & 13.5 & 27.0 & $\mathrm{~L} / \mathrm{I}$ & 119 & 96.0 & & 96.0 & 3.1 \\
\hline \multirow{4}{*}{ [26] } & 7 & 6.5 & 45.5 & I & 48 & 97.4 & & 97.4 & 4.9 \\
\hline & 7 & 70 & 100 & L & 26 & 100.0 & & 100.0 & 6.6 \\
\hline & t & 1.0 & 4.0 & I & 52 & 88.9 & & 88.9 & 4.7 \\
\hline & 9 & 6.0 & 54.0 & I & 39 & 100.0 & & 100.0 & 5.4 \\
\hline
\end{tabular}

values used in our analysis are those shown in the last two columns in Table 1.

2. Values of BC were sampled for each clinical study analyzed according to the corresponding Gaussian distributions $N[\mu, \sigma]$, and the function in equation (5) was fitted to the set of values obtained (or subsets characterized by specific fractionation schemes) using the Levenberg-Marquardt method [29]. As a result, the values of $D_{50}, \gamma$, and $\alpha / \beta$ were found. In this process, the distributions were truncated to avoid BC values above 100 .

It must be indicated that, in case of asymmetric CI, it is possible to consider other distributions for BC. However, as it has been shown in [30], they produce equivalent results.

3. Step 2 was repeated 10,000 times, thus providing the statistics required to determine the distributions of $D_{50}$, $\gamma$, and $\alpha / \beta$. For each of them, the median and the $95 \%$ $\mathrm{CI}$ were calculated. The uncertainties quoted in this work correspond to these CIs, which are indicated between square parentheses accompanying the average values of the corresponding quantity.

To check the convergence of procedure, fits in which $\alpha / \beta$ was fixed to a given value were performed. In these fits, $D_{50}$ and $\gamma$ were obtained as well as their $\chi^{2}$ per degree of freedom, $\chi^{2} / v$.

\section{Results}

We used equation (4) to calculate $\mathrm{EQD}_{2}$ corresponding to the fractionation schemes analyzed, with $\alpha / \beta$ $=1.5$ and 3.0 Gy as the values usually employed in the last years for comparing different fractionation schemes 


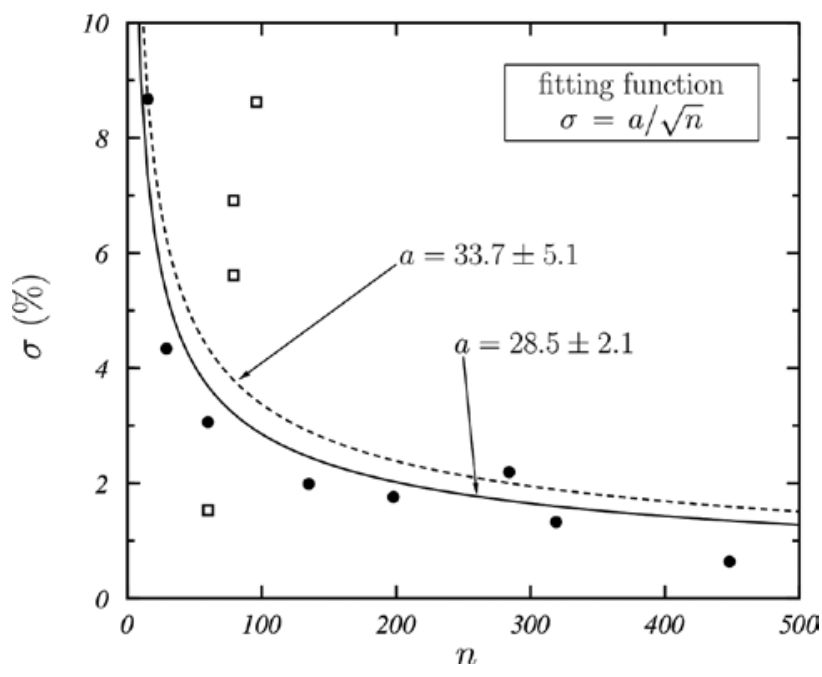

Fig. 1. $\sigma$, defined in equation (6) vs. the number of patients $\mathrm{n}$ of clinical studies with $\mathrm{CI}$ available. The curves are fits of the function $\sigma=a n^{-\frac{1}{2}}$ to all data (dashed curve) and to data shown with solid circles (solid curve), excluding the outliers (open squares). The values of the fitting parameter a in both cases are given

in prostate cancer. BC at 5 years of the various clinical studies are shown in Figure 2, as a function of $\mathrm{EQD}_{2}$ and grouped according to their fraction number.

No correlation among the various fractionation schemes was observed, particularly for the two single fraction schemes included (red open squares). This may be due to the fact that the $\alpha / \beta$ considered could be incorrect. To investigate whether this is the case, we analyzed the data by using the Monte Carlo procedure described above. The results obtained for the medians and 95\% CI of distributions of the fitting parameters are shown in Figure 3, with dose $d_{90 \%}$ additionally given. This is the dose required to produce, in a single fraction scheme, $\mathrm{BC}=90 \%$ at 5 years, which is approximately the average $\mathrm{BC}$ value found for all the studies analyzed.

Three datasets were studied. The first one included all the schemes shown in Table 1. The other two corresponded to more restricted regions of dose per fraction, and were considered to study whether the fitting parameters change significantly. We analyzed $d \geq 9.5 \mathrm{~Gy}$, choosing large doses per fraction, where the failure of LQ model has been suggested (e.g. [16]), and $d \leq 11.5 \mathrm{~Gy}$, neglecting the highest doses per fraction and, in particular, the only two studies with a single dose. The curves obtained with fitting parameters determined in the Monte Carlo analysis are shown with solid lines in Figure 3, together with $\chi^{2} / v$ values of the fits.

Figure 4 shows the results obtained by fitting the function in equation (5) to all data included in Table 1 for fixed values of $\alpha / \beta$. The variation of $D_{50}, \gamma, \chi^{2} / v$, and $d_{90 \%}$ with $\alpha / \beta$ is shown in the several panels. The vertical dotted lines indicate the value $\alpha / \beta=22.8 \mathrm{~Gy}$, where $\chi^{2} / v=1.90$ reaches a minimum that corresponds to $D_{50}=27.7 \mathrm{~Gy}, \gamma=1.45$, and $d_{90 \%}=22.3 \mathrm{~Gy}$. In Figure 2C, we show BC at 5 years of the various clinical studies analyzed as a function of $\mathrm{EQD}_{2}$, grouped according to their fraction number for this value of the $\alpha / \beta$ parameter.

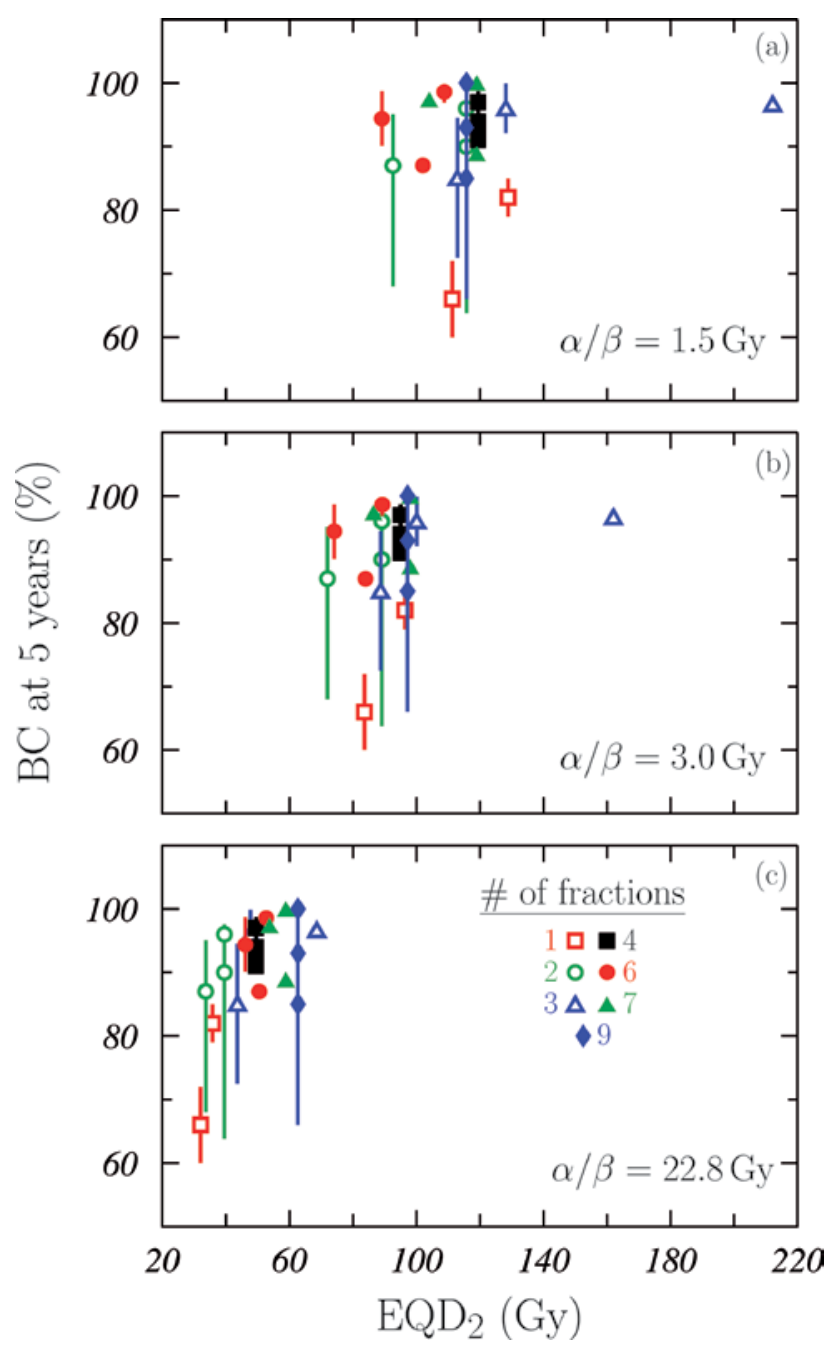

Fig. 2. $\mathrm{BC}$ at 5 years vs. $\mathrm{EQD}_{2}$ calculated using equation (4) with $\alpha / \beta=1.5$ Gy (a), 3.0 Gy (b), and 22.8 Gy (c). Data are grouped according to the number of fractions

These results are consistent with those obtained in the Monte Carlo analysis where, for the three datasets considered, a high value of $\alpha / \beta$ and above $18 \mathrm{~Gy}$ was found. The situation observed for $\chi^{2} / v$ in Figure $4 C$ is very indicative: after a rapid reduction and once the minimum is reached, $\chi^{2} / v$ remains almost constant with increasing $\alpha / \beta$.

\section{Discussion}

The analysis of clinical studies about monotherapy HDR-BT in prostate cancer indicate that high $\alpha / \beta$ values are required to describe adequately the data available. As shown in Figure 3C, this occurs even if only the data with doses per fraction $6 \mathrm{~Gy}<d \leq 11.5 \mathrm{~Gy}$ are considered and the schemes with a single fraction are excluded.

When all data are included, the single fraction schedule producing $\mathrm{BC}=90 \%$ is $d_{90 \%}=22.3 \mathrm{~Gy}[21.5,24.2 \mathrm{~Gy}]$, which corresponds to $\mathrm{EQD}_{2}=40.6 \mathrm{~Gy}[32.1,46.6 \mathrm{~Gy}]$. In this case, a single fraction of $19 \mathrm{~Gy}$ used in a study by Prada et al. [13] and corresponding to $\mathrm{EQD}_{2}=32.0 \mathrm{~Gy}$ would 

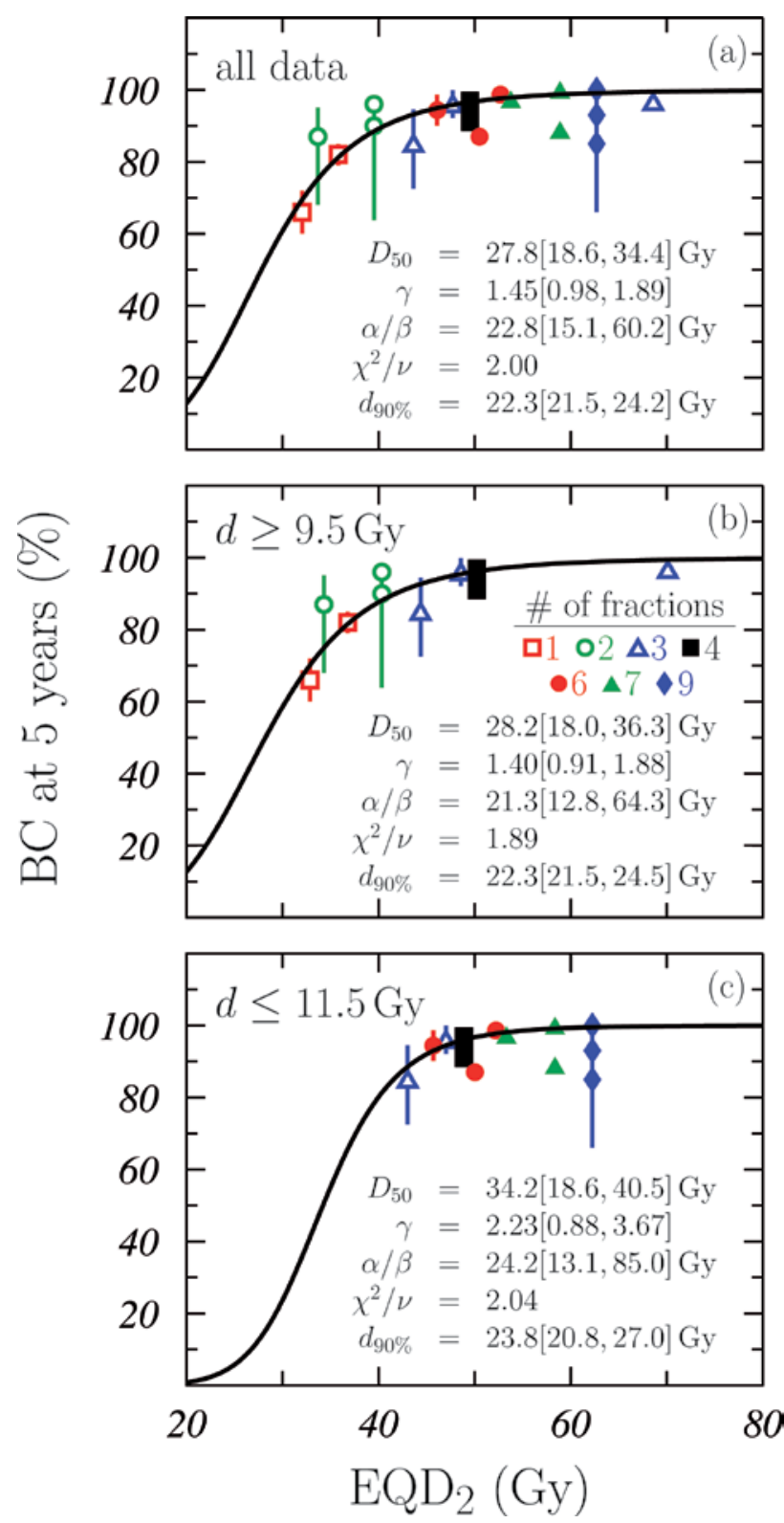

Fig. 3. $\mathrm{BC}$ data vs. $\mathrm{EQD}_{2}$ grouped according to the number of fractions. The results in the inset tables are the medians and $95 \% \mathrm{CI}$ of the fitting parameter distributions obtained in the Monte Carlo procedure. Solid curves represent the function in equation (5) obtained for the medians of the Monte Carlo fitting parameters. The $\chi^{2} / v$ for these fitting curves are given. A) All the data in Table 1 were included in the Monte Carlo analysis. B, C) Only the data with $d \geq 9.5$ Gy and $d \leq 11.5$ Gy, respectively, were included. Finally, the $\mathrm{d}_{90 \%}$ values are also shown

produce $\mathrm{BC}=69.6 \%[64.8,74.6 \%]$, close to the value of $\mathrm{BC}$ $=66 \%[60,72 \%]$ quoted by these authors, and much smaller than the prediction of Mavroidis et al. who estimated BC above $95 \%$ for a single fraction between 19.2 and $19.7 \mathrm{~Gy}$ [9]. On the other hand, we found $\mathrm{BC}=81.4 \%$ [78.6, $84.0 \%$ ] for a single fraction of $20.5 \mathrm{~Gy}$, for which Prada et al. obtained $82 \%$ [79.0, 85.0\%] [14].
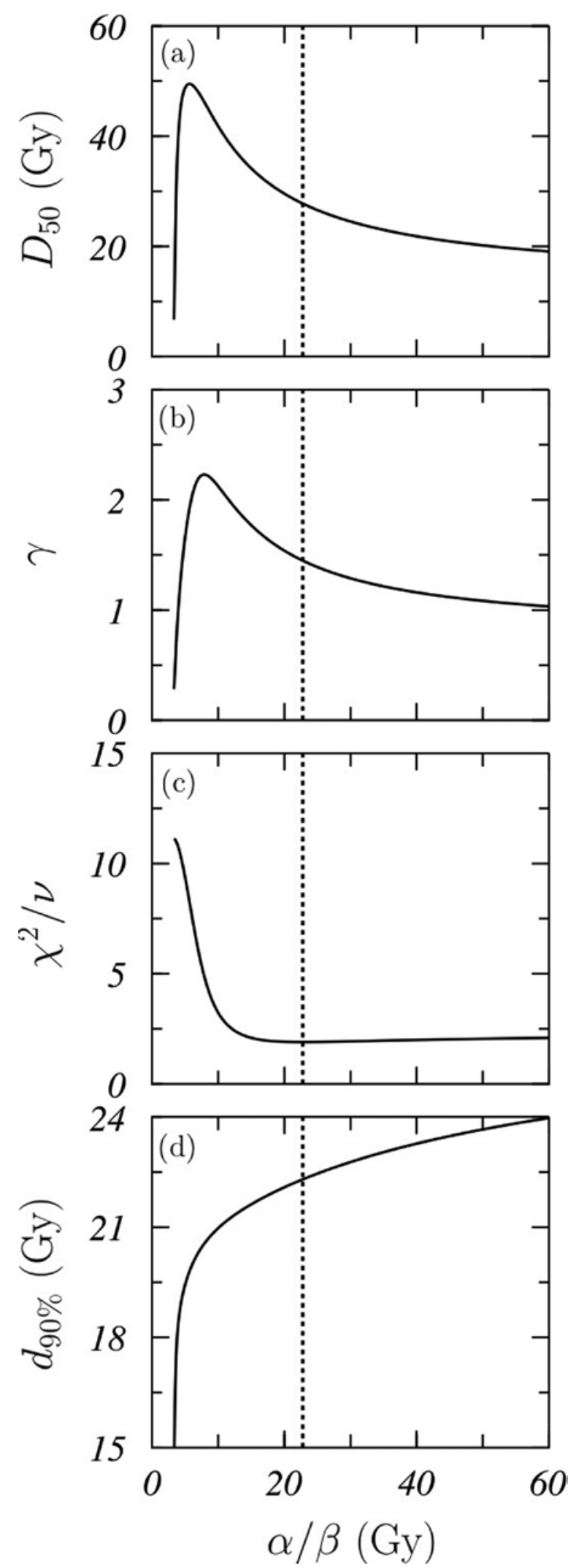

Fig. 4. Dependence of (A) $D_{50},(\mathbf{B}) \gamma,(\mathbf{C}) \chi^{2} / v$, and (D) $d_{90 \%}$ with $\alpha / \beta$. For each value of $\alpha / \beta$, a fit of the function in equation (5) to all the data considered in the present analysis was performed. Vertical dotted lines correspond to $\alpha / \beta=22.8 \mathrm{~Gy}$, where $\chi^{2} / v$ reaches a minimum 
Table 2. Dose per fraction needed to reach BC values of 90 and $95 \%$ according to the results of the present work. Results for various schemes including 1 to 4 fractions are presented

$d$ (Gy)

\begin{tabular}{lcccc}
\hline BC & 1 fraction & 2 fractions & 3 fractions & 4 fractions \\
\hline $90 \%$ & $22.3[21.5,24.2]$ & $13.8[13.4,14.0]$ & $10.2[9.6,10.5]$ & $8.1[7.4,8.5]$ \\
\hline $95 \%$ & $24.3[23.0,27.9]$ & $15.1[14.6,16.2]$ & $11.2[10.9,11.6]$ & $9.0[8.6,9.3]$
\end{tabular}

The lower $\alpha / \beta$ average obtained in the three data subsets analyzed was 21.3 Gy [12.8, 64.3 Gy] (see Figure 3B), well above 1.5 and 3.0 Gy values usually considered (Figure 2). Our results indicate low sensitivity of prostate cancer to fractionation with high doses per fraction, much lower than that indicated by the small $\alpha / \beta$ mentioned above. This has been claimed by other authors, highlighting that the design of hypofractionated schemes for prostate cancer radiotherapy need to be proceed with caution [8].

It is worth noting that LQ model results are fully correct only in the dose per fraction region where its defining parameters were fitted. Low values of $\alpha / \beta$ are consistently obtained in studies where low doses per fraction are used [31]. However, some authors have observed that the LQ model using these low values of $\alpha / \beta$ for high doses per fraction, produces local control levels below expectations [32], which is in agreement with our findings. Therefore, we can consider the LQ model as a valid approximation in a restricted range of dose per fraction, whereas a pragmatic solution recently proposed for cervix cancer [17] can be adopted using an $\alpha / \beta$ value when one compares fractionations in which low doses per fraction are given, and a different one to evaluate schedules in the high-dose per fraction region.

This high $\alpha / \beta$ explains why the single fraction schemes have not produced the clinical results expected from the LQ model, when the $\alpha / \beta$ determined for low doses per fraction is considered [13,14]. In this case, relatively high $\mathrm{EQD}_{2}$, calculated with low values of $\alpha / \beta$, produce modest BC probabilities (see Figure 2).

In this respect, it is worth mentioning that three studies were not included in our analysis because they do not match the selection criteria. The first one is that of Hoskin et al. [33], who quoted $\mathrm{BC}=94 \%$ for treatments with a single fraction of 19 or $20 \mathrm{~Gy}$ for 49 months of follow-up for 49 patients having intermediate- or high-risk disease. These $B C$ values are similar to those shown by other fractionation schemes, with high control rates confirmed after completion of patient's follow-up (as the authors themselves indicate). Moreover, a larger patient sample would be desirable.

In the second study, Mendez et al. [34] indicated that the biochemical failure rate found at 30 months was around $10 \%$, which is larger than expected for a single fraction of $19 \mathrm{~Gy}$.

Finally, Barnes et al. [35] included 28 patients with low- and intermediate-risk, and observed $80.6 \%$ biochemical failure-free survival after 3 years. This value was qualified by the authors as a suboptimal biochemical control.

The estimated $D_{50}$ is significantly smaller than those of classical schemes. For the whole sample of studies, we found $D_{50}=27.8 \mathrm{~Gy}$, while Trada et al. obtained 39.8 and $45.1 \mathrm{~Gy}$, respectively, for low- and intermediate-risk patients, after analyzing various clinical studies of prostate cancer EBRT, with fractions between 1.8 and 2.0 Gy [36]. This may be due (at least to some extent) to the shortening in the overall treatment time in HDR-BT schemes with respect to EBRT, which could produce an increase of the treatment effect for low- and intermediate-risk patients [37].

With regards to $\gamma$, the value obtained for the whole set of clinical studies analyzed was $\gamma=1.45[0.98,1.89]$. Trada et al., in the same revision above mentioned, quoted average $\gamma$ values between 1.2 and 3.2 [36]. In a similar work, in which studies using hypofractionation were excluded, Diez et al. [38] found $\gamma=1.78$ [0.54, 3.02] for lowrisk patients and $\gamma=1.63[1.13,2.14]$ for intermediate- and high-risk patients. Even though these values are compatible with ours, it is worth noting that the non-randomized studies analyzed by Diez et al. defined biochemical failure according to ASTRO criterium instead of that used in our study.

Our results show that doses used in a single fraction must be above 22 Gy to obtain BC $\geq 90 \%$ and above 24 Gy for $\mathrm{BC} \geq 95 \%$. A single fraction of $22-24 \mathrm{~Gy}$ may compromise the toxicity for normal tissues, and it would be interesting to analyze this aspect with a methodology similar to that used in our study, including possible changes in the $\alpha / \beta$ of normal tissues for high doses per fraction. In any case, it is interesting to consider other fractionation alternatives. The results are shown in Table 2. For 3 and 4 fraction schemes, doses per fraction similar to those already utilized in some of the studies shown in Table 1 were found. The only exception was that of Kukiełka et al., who used 3 fractions of 15 Gy [22]; however, according to our calculations, it is not necessary to reach such high values to achieve adequate BC. For 2 fraction schemes and $\mathrm{BC}=90 \%$, we found that fraction doses of approx. 13.5 Gy are necessary, a value similar to that considered in references [12,25].

A fractionated treatment can be analyzed in terms of BED. For a single fraction of $22.3 \mathrm{~Gy}$ and assuming $\alpha / \beta$ $=22.8 \mathrm{~Gy}$, we found BED $=44.1 \mathrm{~Gy}$ for $\mathrm{BC}=90 \%$ (see eq. (2)), much smaller than those obtained, assuming the low $\alpha / \beta$ values currently adopted (188.1 Gy and 353.8 Gy for $\alpha / \beta$ equal to $3 \mathrm{~Gy}$ and $1.5 \mathrm{~Gy}$, respectively). $\alpha / \beta$ and BED can be considered as mutually dependent quantities that must be chosen according to the specific treatment and fractionation. Reference BED values must be used carefully considering the clinical context, for which they were estimated. For example, Zaorsky et al. [39] used the same $\alpha / \beta$ for EBRT, with doses per fraction relatively low, and HDR-BT. These authors concluded that in- 
creasing the BED obtained for $\alpha / \beta=1.5$ Gy above $200 \mathrm{~Gy}$ does not produce any effect. However, the BED may be wrongly calculated for HDR-BT because of the very low $\alpha / \beta$ value considered. In the same way, as can be seen in Figure 2, the value of $\mathrm{EQD}_{2}$ depends on the value of $\alpha / \beta$ considered to calculate it. Therefore, the $\mathrm{EQD}_{2}$ values estimated for the set of treatments studied in this work are valid only in the specific range of doses per fraction analyzed.

The results presented in this work have some limitations: 1 . The dataset fulfilling the criteria imposed is small and is affected by uncertainties that are considerably large; 2 . There is some heterogeneity among treatment techniques employed in the different studies; 3 . The two single fraction studies included in our analysis belong to the same institution. In addition, the use of hormonal therapy, the implant technique, the heterogeneity of absorbed dose, different criteria for prescribed dose used by various institutions, limitations imposed on dose that normal tissues may receive, uncertainties due to the presence of low and intermediate patients in the cohort analyzed, etc., are the conditions that may modify the value of the fitted parameters.

\section{Conclusions}

The $\alpha / \beta$ value of 22.8 Gy found in our analysis, with a range of dose per fraction between 6.0 and $20.5 \mathrm{~Gy}$, is much greater than $1.5 \mathrm{~Gy}$ or 3.0 Gy currently considered for prostate cancer radiotherapy with low doses per fraction. This high value of $\alpha / \beta$ explains reasonably well the data available in the region of the high doses per fraction considered. The absorbed dose in monotherapy HDR-BT, in a single fraction scheme, allowing to obtain BC $=90 \%$ and $95 \%$ are 22 Gy and $24 \mathrm{~Gy}$, respectively.

The limitations of our analysis, such as the strong influence of single fraction schemes in the fitting procedure or the heterogeneity of data, can be overcame by considering additional clinical studies.

\section{Funding}

This work was partially supported by the Spanish Ministerio de Ciencia y Competitividad (FPA201567694-P), the European Regional Development Fund (ERDF), and the Junta de Andalucía (FQM0387).

\section{Conflict of interest}

The authors report no conflict of interest.

\section{References}

1. Yoshioka Y, Nose T, Yoshida K et al. High-dose-rate interstitial brachytherapy as a monotherapy for localized prostate cancer: treatment description and preliminary results of a phase I/II clinical trial. Int J Radiat Oncol Biol Phys 2000; 48: 675-681.

2. Demanes DJ, Rodriguez RR, Altieri GA. High dose rate prostate brachytherapy: the California Endocurietherapy (CET) method. Radiother Oncol 2000; 57: 289-296.

3. Martinez AA, Pataki I, Edmundson G et al. Phase II prospective study of the use of conformal high-dose-rate brachyther- apy as monotherapy for the treatment of favorable stage prostate cancer: a feasibility report. Int J Radiat Oncol Biol Phys 2001; 49: 61-69.

4. Yoshioka Y, Suzuki O, Otani Y et al. High-dose-rate brachytherapy as monotherapy for prostate cancer: technique, rationale and perspective. I Contemp Brachytherapy 2014; 6: 91-98.

5. Demanes DJ, Ghilezan MI. High-dose-rate brachytherapy as monotherapy for prostate cancer. Brachytherapy 2014; 13: 529-541.

6. Vogelius IR, Bentzen SM. Meta-analysis of the alpha/ beta ratio for prostate cancer in the presence of an overall time factor: bad news, good news, or no news? Int I Radiat Oncol Biol Phys 2013; 85: 89-94.

7. Koontz BF, Bossi A, Cozzarini C et al. A systematic review of hypofractionation for primary management of prostate cancer. Eur Urol 2015; 68: 683-691.

8. Höcht S, Aebersold DM, Albrecht C et al. Hypofractionated radiotherapy for localized prostate cancer. Strahlenther Onkol 2017; 193: 1-12.

9. Mavroidis P, Milickovic N, Cruz WF et al. Comparison of different fractionation schedules toward a single fraction in high-dose-rate brachytherapy as monotherapy for low-risk prostate cancer using 3-dimensional radiobiological models. Int J Radiat Oncol Biol Phys 2014; 88: 216-223.

10. Martinez AA, Demanes J, Vargas C et al. High-dose-rate prostate brachytherapy: an excellent accelerated-hypofractionated treatment for favorable prostate cancer. Am J Clin Oncol 2010; 33: 481-488.

11. Tselis N, Tunn UW, Chatzikonstantinou G et al. High dose rate brachytherapy as monotherapy for localised prostate cancer: a hypofractionated two-implant approach in 351 consecutive patients. Radiat Oncol 2013; 8: 115.

12. Jawad MS, Dilworth JT, Gustafson GS et al. Outcomes associated with 3 treatment schedules of high-dose-rate brachytherapy monotherapy for favorable-risk prostate cancer. Int J Radiat Oncol Biol Phys 2016; 94: 657-666.

13. Prada PJ, Cardenal J, Blanco AG et al. High-dose-rate interstitial brachytherapy as monotherapy in one fraction for the treatment of favorable stage prostate cancer: Toxicity and long-term biochemical results. Radiother Oncol 2016; 119: 411-416.

14. Prada PJ, Ferri M, Cardenal J et al. High-dose-rate interstitial brachytherapy as monotherapy in one fraction of $20.5 \mathrm{~Gy}$ for the treatment of localized prostate cancer: Toxicity and 6-year biochemical results. Brachytherapy 2018; 18: 845-851.

15. Guerrero M, Li XA. Extending the linear-quadratic model for large fraction doses pertinent to stereotactic radiotherapy. Phys Med Biol 2004; 49: 4825-4835.

16. Nahum AE. The radiobiology of hypofractionation. Clin Oncol (R Coll Radiol) 2015; 27: 260-269.

17. Tornero-López AM, Guirado D. Radiobiological considerations in combining doses from external beam radiotherapy and brachytherapy for cervical cancer. Rep Pract Oncol Radiother 2018; 23: 562-573.

18. Roach M, Hanks G, Thames H Jr et al. Defining biochemical failure following radiotherapy with or without hormonal therapy in men with clinically localized prostate cancer: recommendations of the RTOG-ASTRO Phoenix Consensus Conference. Int J Radiat Oncol Biol Phys 2006; 65: 965-974.

19. Yoshioka Y, Konishi K, Sumida I et al. Monotherapeutic high-dose-rate brachytherapy for prostate cancer: five-year results of an extreme hypofractionation regimen with 54 Gy in nine fractions. Int J Radiat Oncol Biol Phys 2011; 80: 469-475.

20. Rogers CL, Alder SC, Rogers RL et al. High dose brachytherapy as monotherapy for intermediate risk prostate cancer. J Urol 2012; 187: 109-116. 
21. Barkati M, Williams SG, Foroudi F et al. High-dose-rate brachytherapy as a monotherapy for favorable-risk prostate cancer: a Phase II trial. Int J Radiat Oncol Biol Phys 2012; 82: 1889-1896.

22. Kukiełka AM, Dąbrowski T, Walasek T et al. High-dose-rate brachytherapy as a monotherapy for prostate cancer - single-institution results of the extreme fractionation regimen. Brachytherapy 2015; 14: 359-365.

23. Hauswald H, Kamrava MR, Fallon JM et al. High-dose-rate monotherapy for localized prostate cancer: 10-year results. Int J Radiat Oncol Biol Phys 2016; 94: 667-674.

24. Strouthos I, Tselis N, Chatzikonstantinou G et al. High dose rate brachytherapy as monotherapy for localised prostate cancer. Radiother Oncol 2018; 126: 270-277.

25. Nagore G, Lopez Guerra JL, Krumina E et al. High dose rate brachytherapy for prostate cancer: A prospective toxicity evaluation of a one day schedule including two 13.5 Gy fractions. Radiother Oncol 2018; 127: 219-224.

26. Yamazaki H, Masui K, Suzuki G et al. Comparison of three moderate fractionated schedules employed in high-dose-rate brachytherapy monotherapy for clinically localized prostate cancer. Radiother Oncol 2018; 129: 370-376.

27. Källman P, Agren A, Brahme A. Tumour and normal tissue responses to fractionated non uniform dose delivery. Int $\mathrm{J} R \mathrm{R}$ diat Biol 1992; 62: 249-262.

28. Joiner MC, Bentzen SM. Fractionation: the linear-quadratic approach. In: Joiner MC, van der Kogel A (eds.). Basic clinical radiobiology. 4th ed. CRC Press, Boca Raton 2009; 102-119.

29. Press WH, Teukolsky SA, Vetterling WT et al. Numerical recipes in Fortran 90. $2^{\text {nd }}$ ed. Cambridge University Press 2002.

30. Guirado D, De la Vega JM, Guerrero R, Lallena AM. Radiobiological parameters for breast cancer: a Monte Carlo analysis of START trial published results. Br J Radiol 2018; 91: 20170979.

31. Miralbell R, Roberts SA, Zubizarreta E, Hendry JH. Dose-fractionation sensitivity of prostate cancer deduced from radiotherapy outcomes of 5,969 patients in seven international institutional datasets: $\alpha / \beta=1.4(0.9-2.2)$ Gy. Int J Radiat Oncol Biol Phys 2012; 82: e17-24.

32. Roberts SA, Miralbell R, Zubizarreta EH et al. A modelled comparison of prostate cancer control rates after high-doserate brachytherapy (3145 multicentre patients) combined with, or in contrast to, external-beam radiotherapy. Radiother Oncol 2014; 111: 114-119.

33. Hoskin P, Rojas A, Ostler P et al. Single-dose high-dose-rate brachytherapy compared to two and three fractions for locally advanced prostate cancer. Radiother Oncol 2017; 124: 56-60.

34. Mendez LC, Ravi A, Chung $\mathrm{H}$ et al. Pattern of relapse and dose received by the recurrent intraprostatic nodule in lowto intermediate-risk prostate cancer treated with single fraction 19 Gy high-dose-rate brachytherapy. Brachytherapy 2018; 17: 291-297.

35. Barnes JM, Gabani P, Sanders M et al. Single fraction highdose-rate brachytherapy as monotherapy for low and intermediate risk prostate cancer: toxicities and early outcomes from a single institutional experience. J Contemp Brachytherapy 2019; 11: 399-408.

36. Trada Y, Plank A, Martin J. Defining a dose-response relationship for prostate external beam radiotherapy. J Med Imaging Radiat Oncol 2013; 57: 237-246.

37. Thames HD, Kuban D, Levy LB et al. The role of overall treatment time in the outcome of radiotherapy of prostate cancer: an analysis of biochemical failure in 4839 men treated between 1987 and 1995. Radiother Oncol 2010; 96: 6-12.

38. Diez P, Vogelius IS, Bentzen SM. A new method for synthesizing radiation dose-response data from multiple trials ap- plied to prostate cancer. Int J Radiat Oncol Biol Phys 2010; 77: 1066-1071.

39. Zaorsky NG, Palmer JD, Hurwitz MD et al. What is the ideal radiotherapy dose to treat prostate cancer? A meta-analysis of biologically equivalent dose escalation. Radiother Oncol 2015; 115: 295-300. 